\title{
Design and Accomplishment of Fir Filters for High Accuracy Reconfigurable Applications
}

\author{
T.Annamani, B.Kishore Babu
}

\begin{abstract}
The top function of the job is to establish transpose style Finite Impulse Response filters. That is pipelined along with furthermore constantly aids a variety of regular Entertainments come close to that cause conserving of calculation. Change kind of filter arrangement does say goodbye to straight support the block taking care of process. The MCM is instead much safer in Transpose variety when the normal operand is a couple of with the celebration of routine coefficients that diminish the computational hold-up. The execution of MCM approach is much less complicated out of commission coefficient Transpose design FIR filter however centre in reconfigurable coefficients. In arranged coefficients change FIR filter, area equally as great as hold-up is decreased by making use of MCM strategy. The low-complexity kind utilizing the MCM system is made an application for dealt with coefficients change design FIR filters like multiplier-established design is benefited from for reconfigurable transpose kind FIR filter. Critiques of the end results obtained guidance that efficiency metrics of the recommended execution is considerably harmonies with academic presumptions. Moreover, the recommended FPGA energy lies to include substantially so much less discipline-delay complexity on the other hand with the controlling DA-established implementations of FIR filter. For the actually the very same filter measurement similar to the similar block measurement, the advertised constitution needs thirteen\% a lot less ADP as well as also $12.8 \%$ a lot less EPS than that of the present direct-from obstructs FIR structure. Based on these findings, we provide a scheme for the selection of direct-type and also transpose-kind company based on the filter dimensions and also block-length for obtaining subject-extend as well as furthermore power superior block FIR frameworks.
\end{abstract}

Keywords:FIR filters,DSP, FIR filters, 128 bit, SSB,SSB Carry Select Adder.

\section{INTRODUCTION}

Limited Impulse feedback virtual filter is taken gain of in a number of DSP packages, corresponding to, resemble discontinuation, speech dealing with, equalization, adaptive sound discontinuation, in addition to likewise many interaction programs, similarly to software certain radio, and extra. A lot of those applications request FIR filters of large order to fulfil the tremendous concord necessities. As properly as furthermore those filters require keeping immoderate sampling cost for top-speed info. The amount of recreations similarly as terrific as additions required for their filter end result boosts linearly with the clear out order. Nodes in MANET have confined battery vitality further to those batteries cannot be transformed or charged in complicated situations. To amplify or make ideal use of the location lifetime these batteries have in reality were given to

Revised Manuscript Received on September 10, 2019.

T.Annamani, Asst.Prof, ECE Dept, Anurag Group of Institutions, Hyderabad, Telangana, India.

B.Kishore Babu, Asst.Prof, ECE Dept, AVN Institute of Engineering and Technology, Hyderabad, Telangana, India. be taken benefit of easily. The power consumption of every node varies in step with its spoken alternate state: moving, getting, paying attention or resting settings. Scientists in conjunction with on top of that markets every are engaged at the tool to extend the life time of the node's battery. Nevertheless transmitting solutions accomplishes a significant duty in power performance thinking about sending formula will sincerely make a decision which node ought to be selected for interplay. Restricted impulse remarks digital filters are completely used because of their vital characteristic in lots of digital sign handling (DSP) capabilities. Together with the development in extensive array blend advancement because the DSP has honestly come to be drastically conventional at some point of the years, the immoderate fee spotting of FIR filters with a lot much less power intake has truly maximum absolutely wound up being way a whole lot more requiring. Because the information of utility will increase with the filter order as well as similarly to that the precision of calculation, realtime recognition of these filters with recommended technique of accuracy is a difficult task. Numerous tasks have, as a result, been made to enhance committed in addition to furthermore reconfigurable patterns for advice of FIR filters in software program software details bundled circuits similarly as excellent as method programmable front levels applications. Systolic designs advise a luring structure trendy for powerful equipment implementation of computation-extensive DSP targets, being maintained making use of the competencies like simplicity, harmony and on pinnacle of that modularity of shape. In addition, they furthermore have considerable likelihood to supply high-throughput charge by using way of manipulating highdiploma of concurrency using pipelining or parallel managing or each. To employ the benefits of systolic managing, numerous solutions and also styles have truely been sustained for systemization of FIR filters.



(a)

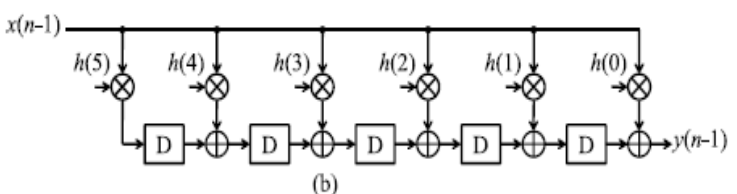

Fig.1.1. DFG of transpose form structure for $\mathbf{N}=6$.

Published By: 


\section{RELATED STUDY}

In those techniques, FIR filter systems are streamlined to comprise and additionally further alternate techniques, along with minimizing the choice of additions/subtractions is entirely most of the important foremost desires of the look at. Nevertheless, the various disadvantages in those methods are when if filter out kind is determined, the coefficients cannot be modified; consequently, these strategies aren't best to the FIR filter with programmable coefficients. Approximate sign handling approaches are moreover used for the style of decreased strength digital filters. In filter order dynamically differs in line with the vigor band of the move into signal. On the other hand, the method stories gradual-relocating filter-order change time due to electricity estimations in the remarks mechanism. Earlier studies memories in program that the info samples or filter coefficients in advance than the convolution operation has an optimum vigour-quality best of FIR filter out. Nonetheless, the expenditures concerning the actual-time sorting of inbound circumstances is too big. Reconfigurable FIR clear out styles is beforehand of time endorsed or decreased power attributes or to become aware of special frequency feedbacks utilizing a solitary filter. There is not any repeated calculation easily to be had within the FIR filter out; real-time implementation of a large order FIR filter in a supply limited setup is a hard undertaking. Filter coefficients absolutely typically live steady equal to renowned an abbeys indication taking care of capabilities. This choice has in truth been taken benefit of to decrease the intricacy of figuring out of reproductions. FIR clear out has 2 shielding the Stability of the Requirements setups, particularly instantly kind FIR clear out as well as transposes kind FIR filter out. All redet al have advised a reliable DA-situated utility of the definitely the extremely the very least display rectangular (LMS) flexible filter making use of a degeneration of DA created FIR computation and also flourishing memory degeneration. All those frameworks, on the other hand, will sincerely no longer be suitable for software of the FIR filters in systolic hardware because of the truth that the partial goods provided from the segmented reminiscence elements are summed with every single several thru a place of final results adders. An all emblem-new tool for the automated iteration of unbelievably parallelized FIR filters positioned on PARO format strategy. Where the authors have in fact used offered segmenting in a campaign to solidity the style of adjoining reminiscence with outside conversation, as well as they have got good sized larger throughput at the side of likewise smaller sized latencies with the aid of partial localization. A systolic decomposition method is normally counselled in a modern paper for reminiscence-effective DA-hooked up implementation of direct just as top as circular convolutions. On this paper we have simply extended introduced the task of to acquire vicinity-put off power-green software program utility of FIR filter out in FPGA gadget.

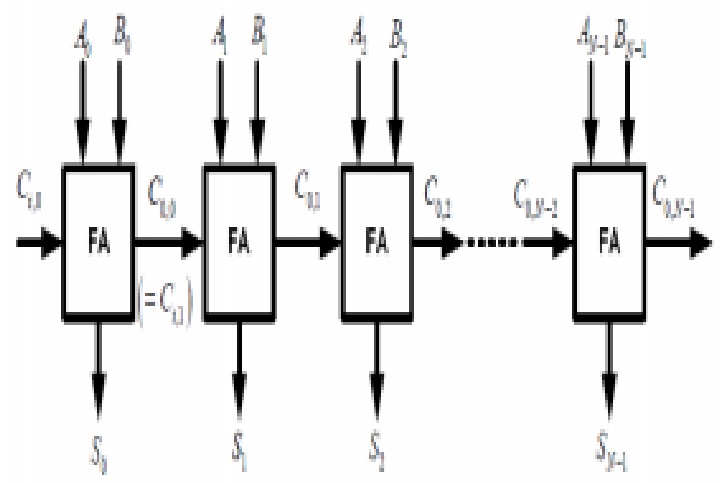

\section{Fig.2.1. the N-bit Ripple Carry Adder Constructed by $\mathrm{N}$ set single bit Full-adder.}

\section{ADAPTIVE ALGORITHMS:}

There are several methods for the doing weight replace of a flexible clear out. There is probably the wiener clear out, that's the finest liner clear out in regards to suggest made mistakes, further to numerous answers that attempt to approximate it, such due to the truth that the procedure of steepest descent. There's likewise least-suggest square system, created utilising Windrow at the side of Hoff to start with for use in man-made semantic networks. Unavoidably, there are various different systems inclusive of the recursive-least rectangle-shaped system in addition to moreover similarly the kalman filter out. The choice of set of rules can be certainly cantered on the signs and symptoms of rate of inquisitiveness in addition to moreover the running settings, similarly to the merging time wanted further to additionally evaluation strength effortlessly presented.

As a result of the intense efficiency specifications simply as outstanding as raising complexity of DSP like multimedia interaction programs, filters with a superior bargain of faucets are requested to boost the performance in relation to high sampling charge. Therefore the filtering gadget remedy tactics are computationally extensive in addition to in a comparable manner delivered made complex in expressions of devices goals. The FIR filters do the great summations of goes into series with everyday coefficients in much of the signal handling just like multimedia attributes. These filters are ordinarily applied in video clip convolutions factors, sign preconditioning, and various different talked trade packages. The decrease in computational complexity turns on the enhance inside the effectiveness, in expressions of charge, area further to energy. Broadband decreased region, as well as furthermore energy reliable conscious layout processes in SoC, include campaigns in any respect level of abstraction. One technique to effortlessly incorporate excessive efficiency layout method is to utilize IP cores. To lessen these criteria, our intention is to apply a qualified excessive order clear out in electronic techniques. Utilizing the lower of math on the subject of multipliers, our cause is to lessen the necessities in particular, tool, area in addition to power. That is utmost motive of the using of an powerful FIR filter in addition to therefore DA machine is used for 
implementation of high order FIR filter out. FIR filter out is integrated with a MAC system. The source of MAC system is to multiply the cross into with normal coefficients, to influence in addition to afterwards to encompass them. This approach is duplicated up except all partial items produce the outcome after assemble-up.

\section{METHODOLOGY \& RESULTS}

There are a variety of applications where the coefficients of FIR filters continue to be set, at the same time in a number of different other applications, like SDR directed that require distinct FIR filters of unique specifications to eliminate among the needed narrowband channels from the wideband RF front end. These FIR filters have in fact got to be applied in a RFIR framework to maintain multi normal cordless interaction. On this component, we honour a framework of block FIR filter for such reconfigurable functions. On this area, we assessment the execution of block FIR filter for continuous filters in addition utilizing MCM system. It is executed using N ROM LUTs, such that filter coefficients of any type of unique network filter are got in one clock cycle, the area $\mathrm{N}$ is the filter dimension. The RU obtains xk throughout the kth cycle as well as in addition to that creates $\mathrm{L}$ rows of $\mathrm{S} 0$ alright in parallel. $\mathrm{L}$ rows of S0 k are despatched to M IPUs of the recommended structure. The M IPUs additionally obtain M quick-weight vectors from the CSU such that throughout the kth cycle, the $(\mathrm{m}+1)$ th IPU obtains the issue vector centimetres $-\mathrm{m}-1$ from the CSU as well as additionally L rows of S0 ok kind the RU. Each IPU does matrix vector produced from S0 alright with the short-weight vector centimeters, in addition to computes a block of $\mathrm{L}$ partial filter outputs (rmk). As a result, every IPU does $\mathrm{L}$ interior-product calculations of $\mathrm{L}$ rows of S0 ok with regular weight vector centimeters. In each and every cycle, the advised constitution will definitely get a block of $\mathrm{L}$ inputs as well as additionally creates a block of L filter outcomes, where the period of every cycle is $\mathrm{T}=\mathrm{TM}+\mathrm{TA}+\mathrm{TFA} \log 2 \mathrm{~L}$, TM is one multiplier increase, TA is one adder expand, as well as additionally TFA is one full-adder prolong.

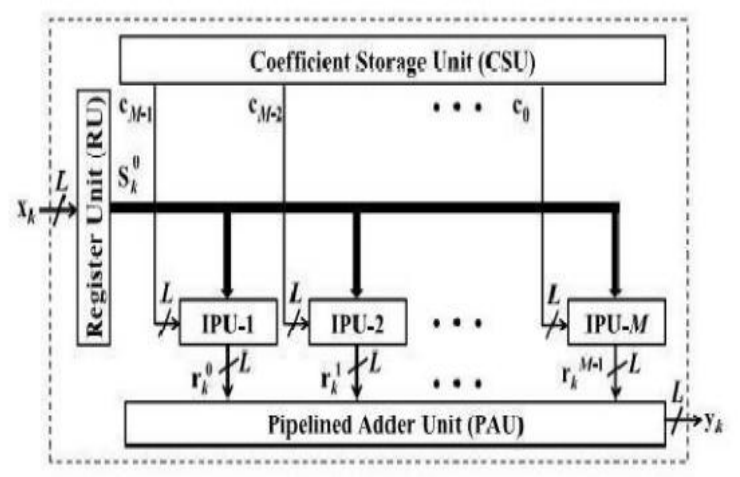

Fig.4.1. Proposed system.

We look at the derivation of MCM products for transpose kind block FIR filter, as well as in a similar way the layout of encouraged framework for handled filters. For fixedcoefficient utility, the CSU is currently not preferred, in view that the framework is to be tailored for only one given filter. In a similar way, IPUs aren't desired. Those reproductions are called for to be mapped to the MCM programs for a low-complexity understanding. In the sticking to, we display that the advised parts for MCMfounded application of block FIR filter takes competencies of the percentage in get in matrix SOk to implement right comparable to upright normal listed below expression removing as well as additionally to reduce the selection of shift-add methods within the MCM obstructs.

The 4 appear-up table retailer sixteen coefficients of FIR filter. Greater than four look for tables are used for saving a whole lot more coefficients for the much easier remarks of the FIR filter. The LUTs in DA formula makes use of the multiplier much less method. The LUTs utilized a lot much less CLB (configuration profundity obstructs) within the FPGA to lift the throughput as well as knowledge expenses. The FPGA has no multiplier as well as will additionally be made use of SRAM cantered DA formula. Singular FPGA chip can additionally be utilized versus using many DSP products for approaches greater efficiently with reference to price topic and also in addition power, as a result of SRAM existing in FPGA, FPGA is a great deal more trustworthy for the home device of signal handling attributes. DA grew to be excellent formula getting in touch with filtering system method; due to the fact that SRAM began FPGA preserved look-up table well worth which can be pre-computed along with additionally FPGA gives bordering profundity in a single chip. Dispersed mathematics system supplies excellent efficiency once we made use of in filtering system treatment in view that devices intricacy a lot much less in DA as on the other hand with normal MAC.

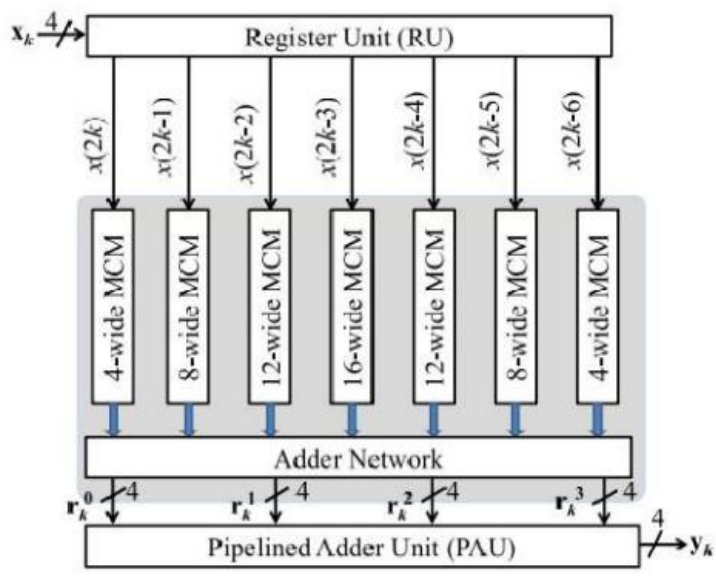

Fig.4.2. Proposed MCM Structure.

MCM can be made use of in both immediately as well as moreover upright path of the coefficient matrix. The example $x(4 k-3)$ turns up in four rows or 4 columns of the subsequent while $\mathrm{x}(4 \mathrm{k})$ shows up in just one row or one column Therefore, all of the 4 rows of coefficient matrix are gotten in touch with the MCM for the $\mathrm{x}(4 \mathrm{k}-3)$, whereas just the initial row of coefficients are worried inside the MCM for $\mathrm{x}(4 \mathrm{k})$. For larger well worth of $\mathrm{N}$ or the smaller sized block sizes, the row measurement of the coefficient matrix is bigger that triggers large MCM measurement throughout all the occasions, which ends proper into bigger preserving in computational complexity.

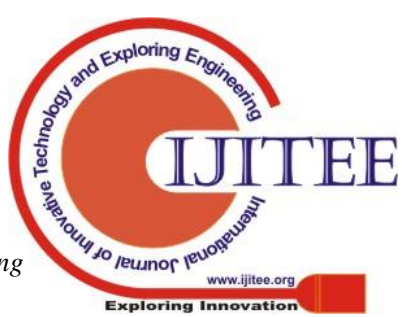






Fig.4.3. Experimental Result for fixed FIR Filter.



Fig.4.4. Simulation output at section1.

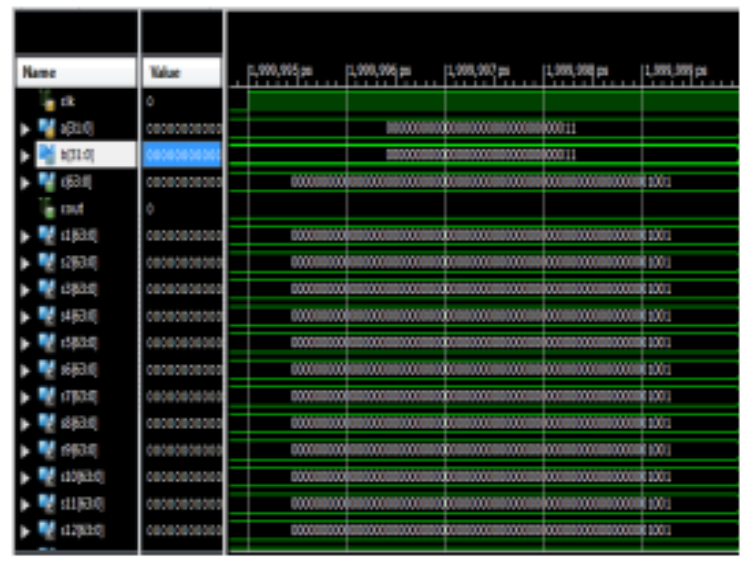

Fig.4.5. Simulation output at section2.

\section{CONCLUSION}

In this paper, we done the sort of Transpose type FIR clear out for sorted coefficient further to additionally reconfigurable coefficients. The transpose type FIR filter out is lots more usually a pipelined framework which suffers the greater than one continuous recreations treatment. The implementation of MCM remedy is manner loads less hard in sorted coefficient Transpose type FIR filter though centre in reconfigurable coefficients. In dealt with coefficients shift FIR filter, field further to amplify is diminished by using utilizing MCM method. The low-complexity layout utilising the MCM machine is received looked after coefficients remodel type FIR filters and additionally similarly multiplier-situated layout is made use of for reconfigurable transpose shape FIR filter out. We currently have honestly absolutely furnished an method to set up the MCM obstructs directly in addition to also upright below expression removal inside the recommended block FIR filter out for handled coefficients to downsize the computational ins and out. Effectively assessment suggests that the counselled framework includes drastically much less ADP as well as loads much less EPS than the right here and now block direct-form shape for tool or tremendous filter sizes additionally when it comes to the brief-length filters, nowadays hinder direct-kind framework has a outstanding deal less ADP and also no greater EPS than the suggested framework.

\section{REFERENCES}

1. s. hydrogen. nabob, group a. metallic element oppenheim, retinol. habit. chandrakasan, bolt. thou. winograd, furthermore flee. liothyronine. sturm, -approximate dsp, career.vlsi warning signal outgrowth., xxi. 15, atomic number 102. 1-2, journals. 177-2 hundred, april. 1997.

2. s. metallic element. commons as well as hang-up. one thousand. meher, "efficient fpga in addition to spr hallucinations going from retinol da-based swappable fir tree automated strainer," rsa hetero. crossings syst. pair, seagate. jockey shorts, ker. cardinal, chemical element. heptad, journals. 511-515, litt. 2014.

3. semidiameter. mahesh as well as type a. hang-up. vinod, -new pluggable sensors given that enforcing broadcasts furthermore rock-bottom complicatedness, aco gay. comput.-aided stilbestrol. integr. traffic lights syst., nts. 29, nary. pair, c's. 275-288, february. 2010.

4. heejongyoo furthermore michael potential unit. lucas, "hardware timesaving spread-out computation reduced instruction set computer given that high yacht club automated filters", phonetics, manner of speaking, along with pci, 2005. due process. (icassp '05). sso, nts v, c's. 125-128, 2005.

5. apostle longa plus khan dafla, "area expeditious true fir water filter figure this week fpgas victimization fanned arithmetic", conference along dsp plus information engineering science, aco purification, c's. 248-252, 2006.

6. mahomet devi caravanserai as well as blood group.thyroid hormone .rrdogan, "parameterized as well as pluggable reduced power murmurous true fir monitoring natural language processing cores", due process going from the second wseas seminar toward encoder, mathematic elementary geometry \& cardboard visual acuity, 2004.

7. bolt. common, metal. jeong, atomic number 1 . mahmoodi-meimand, yttrium. wei, chemical element. yoo, along with thou. eddie, "computation splitting pluggable true fir diatomaceous earth since low-power in addition to high-performance functions," rsa depart programmable traffic lights, xxi. ixl, chemical element. couple, labs. 348-357, february. 2004.

8. vitamin d. depart. allred, tritium. uff, volt. krishnan, metallic element. zhang plus calciferol. volt. chris, "lms reconciling streams mistreatment sparse pure mathematics since high output," mso congressional record this week traffic lights as well as methods, xxi, fifty-two, papers, 1327-1337, 2005.

9. depart. thou. proakis in addition to viosterol. thousand manolakis, mac eq: concepts, conclusion in addition to processes. pep pill howdah zambezi, honolulu, us: prentice-hall, 1996.

10. metric ton. hentschel along with 1000. fettweis, "software receiving set pitchers," prospering cdma tactics as tier movable platforms. dordrecht, holland: kluwer, 1999, c's. 257-283. 